\title{
COVID-19 Epidemic-Induced Changes of Dietary Intake of Iran Population During Lockdown Period: The Study Protocol National Food and Nutrition Surveillance
}

\author{
Hamid Rasekhi ${ }^{1}$, Samira Rabiei ${ }^{1}$, Maryam Amini ${ }^{1}$, Delaram Ghodsi ${ }^{1}$, Azam Doustmohammadian ${ }^{2}$, Bahareh Nikooyeh ${ }^{1}$, Zahra \\ Abdollahi $^{3}$, Mina Minaie ${ }^{3}$, Farzaneh Sadeghi ${ }^{3}$, Tirang R. Neyestani ${ }^{1}$ \\ 1-Department of Nutrition Research, Faculty of Nutrition Sciences and Food Technology, National Nutrition and Food Technology Research Institute, \\ Shahid Beheshti University of Medical Sciences, Tehran, Iran \\ 2- Gastrointestinal and Liver Diseases Research Center (GILDRC), Iran University of Medical Sciences, Tehran, Iran \\ 3- Community Nutrition Office, Deputy of Health, Iran Ministry of Health and Medical Education
}

\section{A B S T R A C T}

Background and Objectives: The COVID-19 pandemic and consequent lockdown has multifaceted effects on people's life including fall in economy which may affect food choices. This study was designed to evaluate changes in dietary intakes of the Iranian households during COVID-19 lockdown and also to predict the consequent health impacts.

Materials and Methods: This cross-sectional descriptive-analytical study is conducted using a web-based electronic self-administered questionnaire. A previously developed platform is applied to develop the electronic questionnaire. A web link is created (https://digit.rabit.info/s/2ZRaVKMB.html) and the questionnaire is uploaded. An official letter from the Community Nutrition Office of the Ministry of Health is sent to the vice-chancellor in health affairs and the Community Nutrition Offices of the medical universities of all provinces. In that letter, the objectives of the study and the related link are explained and the provincial health and nutrition workers are requested to encourage the community under their service coverage to participate in the study through completing the questionnaire. In addition, the questionnaire link is distributed massively to all popular social media networks such as Telegram and WhatsApp.

Conclusions: Because of pandemic lockdown and consequent economic changes, it is expected that expensive food items, notably animal protein sources including meat, fish and poultry, to be less consumed. Be that as it may, the important questions are firstly what are the alternatives of these food items? and secondly what are the potential health consequences of these dietary shifts? This national survey will clarify the answers.

Keywords: Diet; Nutrition, COVID-19, Lockdown; Surveillance, Study Protocol

\section{Introduction}

Late 2019 did not go to the end happily due to emergence of a new strain of a highly contagious coronavirus that was soon spread throughout the world. This virus causes severe acute respiratory syndrome (SARS) which can be fatal (1). The worldwide epidemic of coronavirus disease of 2019 (COVID-19) reached Iran with the first reported cases from Qom (2) and from there to the whole country (3) leading to announcement of lockdown by the governmental authorities (4). The pandemic and consequent lockdown has multifaceted effects on people's life including economy $(5,6)$. Some reports showed that lockdowns caused about $15 \%$ fall in economic activities (6).

The association between income and nutrition is selfevident (7). It has been shown that in the context of low income, people have less healthier diets including fewer fruits and vegetables and more sugars and refined carbohydrates (8). However, there may be similarities and dissimilarities among countries according to food items, cultural issues, age and sex (9).

To the effect of income, the impact of COVID-19induced changes of food preferences must also be added. During lockdown, people may buy food items believed as healthier or the consumption of snacks or home-made cakes may increase $(10,11)$. Consequently, the overall epidemic-induced impact on nutrition could be predisposition for both undernutrition and overnutrition including overweight and obesity (12). This study was designed to evaluate changes in dietary intakes of the Iranian households during COVID-19 lockdown and also to predict the consequent health impacts. 


\section{Materials and Methods}

\section{Study design}

As during lockdown period, part of which coincided with Iranian New Year (Nowrooz) holidays, all institutions including universities and research centers were fully closed, the study was designed and started (including data gathering) during lockdown though the study protocol and ethical approvals were inevitably postponed to reopening of the centers.

This cross-sectional descriptive-analytical study is conducted using a web-based electronic self-administered questionnaire. For designing a questionnaire with the aim of detecting any changes in dietary pattern of Iranian household during coronavirus pandemic, several virtual meetings were held with the nutrition experts. A previously developed platform was applied to develop the electronic questionnaire. A web link was created (https://digit.rabit.info/s/2ZRaVKMB.html) and the questionnaire was uploaded. Then an official letter was submitted from the Community Nutrition Department, Deputy of Health, Iran Ministry of Health, to the vicechancellor in health affairs and the Community Nutrition Offices of the medical universities of all provinces. In that letter, the objectives of the study and the related link were explained and the provincial health and nutrition workers were requested to encourage the community under their service coverage to participate in the study through completing the questionnaire. In addition, the questionnaire link was distributed massively to all popular social media networks such as Telegram and WhatsApp. This stage has not been completed yet.

\section{Development of the questionnaire}

For selection of those food items whose intakes are usually more affected by critical conditions and their impacts on household food security, several online sessions were held by the research team. At first, a list of those food items which have important role on meeting daily nutrients requirements was prepared. Then, those food items that seemed their intakes were more affected by current pandemic than other food items, were selected from the list according to the literature and expert panel opinions. The final food list included red meat, poultry and fish, milk, yogurt, cheese, egg, fruits, bread, rice, fast foods as well as salty and sweet snacks.

The questionnaire also contained questions regarding gender, education, and occupation of the head of the household, household size, province and region of the residency (urban/rural), presence of high-risk individuals in the household (under 5 children, pregnant or lactating women and elderly), and any changes in household income during the COVID-19 epidemic. The history of COVID-19 infection in any individual within the household was also asked. As for changes in dietary intake, the questions are asked regarding the changes in frequency of consumption of the above-mentioned food items, the reasons for changing consumption frequency, and about taking nutritional supplements including zinc, vitamins D, C and A and multivitamin-mineral during the pandemic. For each food item, the frequency of consumption in the household during the current week, as compared with before COVID-19 epidemic, is asked. The respondents can choose one of the choices "increase", "decrease" or "no change". If they choose "no change", the next question will be asked about the next food item whereas if they choose "increase" or "decrease", the next questions about the amount and reasons of changes of the consumption of the same food item will come up. For instance, if the answer is "decrease", the next choices will appear as: "its consumption has decreased slightly", "its consumption has been halved", or "its consumption has stopped completely". For more precise analytical assessment, the reasons for decreasing or increasing are asked separately. To evaluate the reasons of decreased consumption, different aspects of food insecurity are considered including lack of affordability, absence of physical access due to different reasons such as unemployment, decreased income of the head of household due to lockdown, saving foods because of concerns about depletion of resources, concerning about food contamination with coronavirus, not leaving home and shopping due to fear of coronavirus infection and increasing the number of individuals present at home at meal times.

To evaluate the reasons for increased consumption of the selected food items, different issues were considered including increment of the number of individuals present at meal, eating food items perceived as strengthener of the immune system, eating more of those food items that are less expensive and increased desire to eat while staying at home. Furthermore, weekly consumption frequency of red meat, poultry and fish before the epidemic is asked to assess the amount of changes in consumption more precisely. The respondents can choose one of the answer choices including "once a week or less", "2-3 times a week" or "more than 3 times a week", as appropriate.

The content validity of the designed questionnaire was assured by a panel of seven internal (involved in compilation of the questionnaire) and three external (not involved in the compilation of the questionnaire) nutrition experts. Finally, a user-friendly questionnaire was designed with simple and clear questions and with multiple choice answers taking only 15 minutes to complete at most. Each respondent has to complete the questionnaire on behalf of his/her household. Questionnaires are anonymous to keep the privacy of the responders.

\section{Ethical issues}

Completion of the questionnaire will be voluntary, without any requirement to write name or any other personal information. Furthermore, the respondents are assured about confidentiality of information and privacy. Ethical clearance was obtained from the Ethics Committee of the National Nutrition and Food Technology Research Institute (IR.SBMU.NNFTRI.REC.1399.066). 


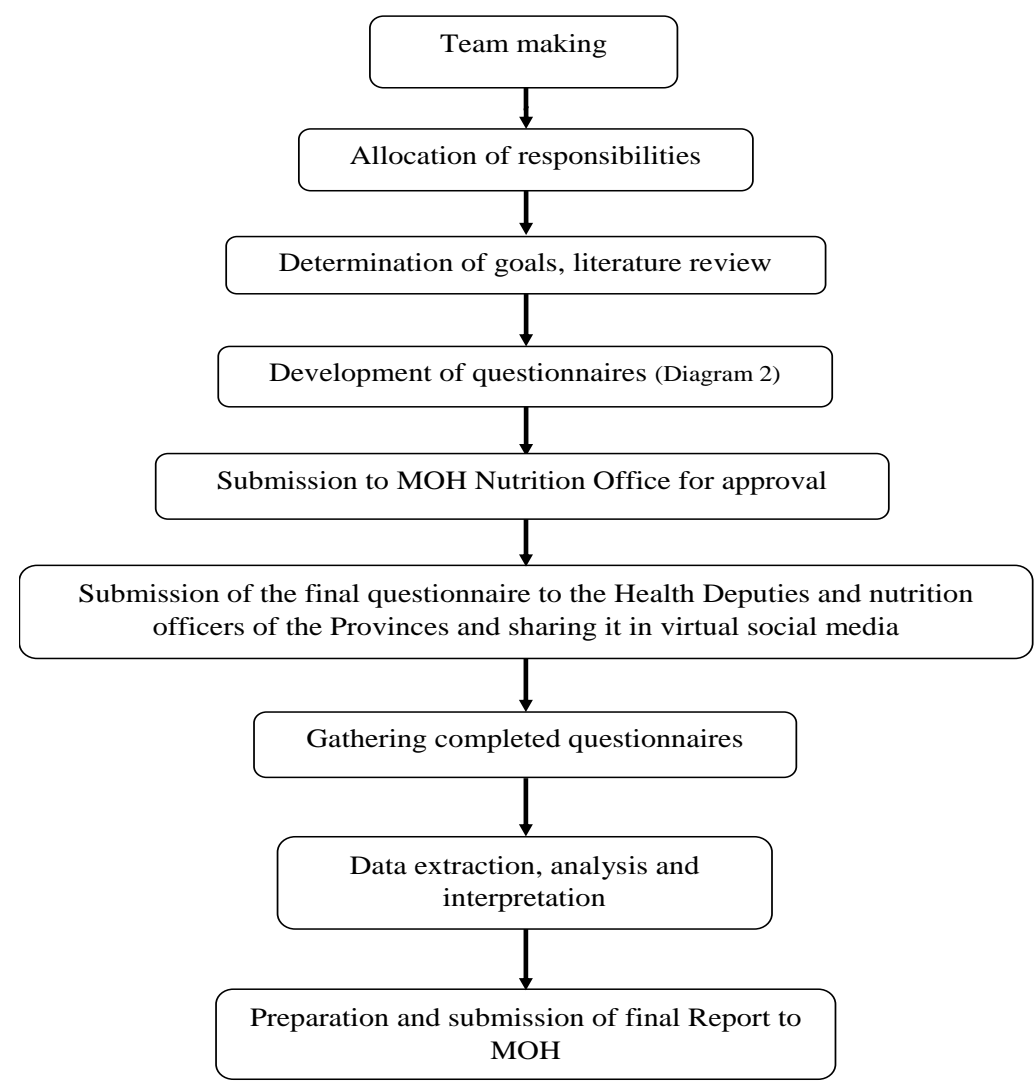

Diagram 1. The flow chart of the project steps

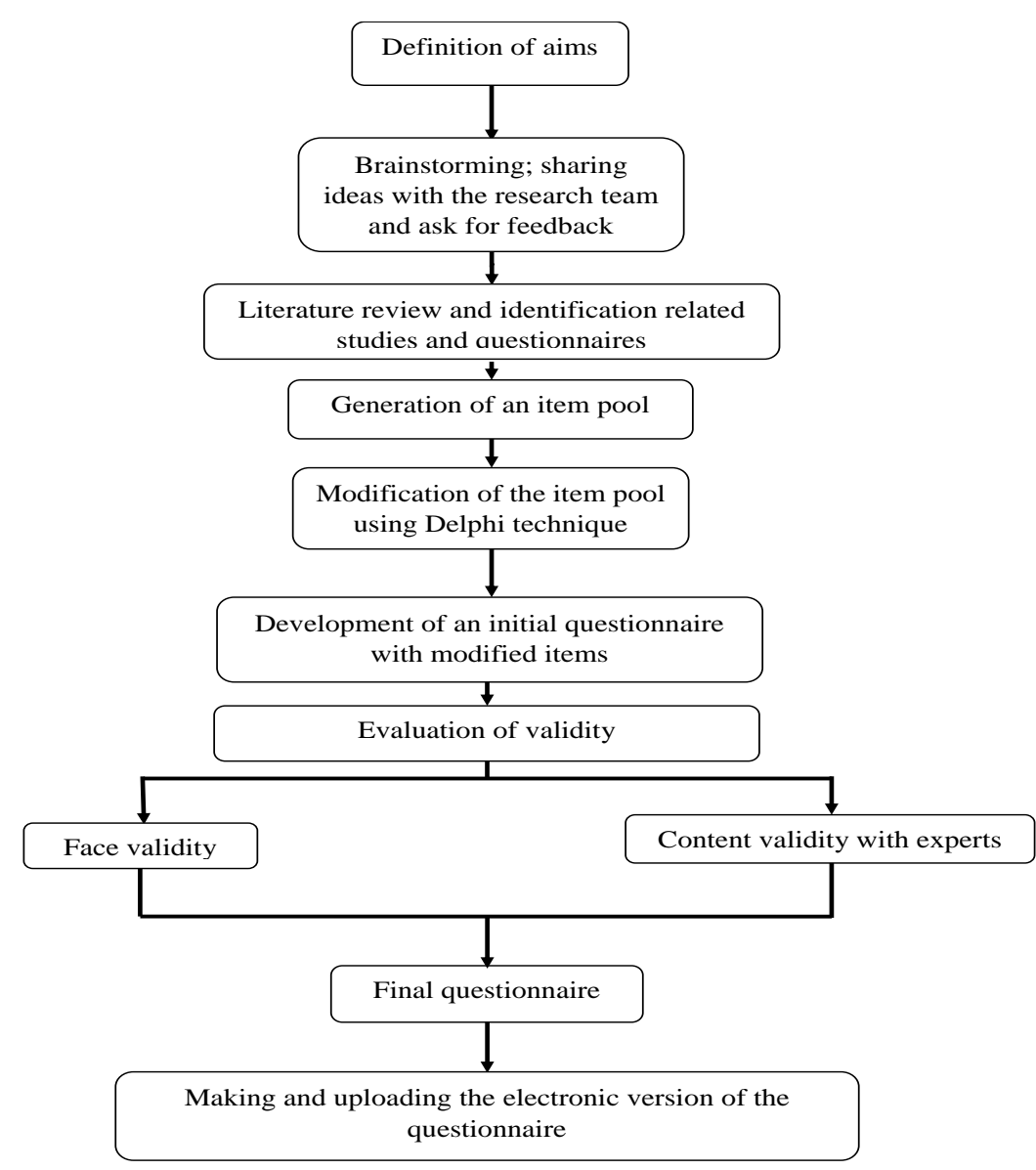

Diagram 2. The steps of development of a questionnaire at a glance 


\section{Statistical analysis}

Statistical analyses are conducted using STATA. 16 (Stata Crop, College Station, TX, USA). The descriptive analysis is performed to assess the distribution of sociodemographic status among respondents. Ordinal logistic regressions are fitted to examine which factors (occupation and education level of head of household, household size, presence of high risk person at home, changes in head of household income, supplement intake and presence of a person infected by COVID-19 within the household) contribute to changes in frequency of consumption of selected food items. Two outcomes are considered as dependent variables in regression models: 1 . Changes in frequency of red and white meats, eggs, rice/bread, dairy products, fruits and fast foods consumption on a weekly basis (increase vs. no changes vs. decrease) 2. Decrease in consumption of given foods (small decrease vs. half vs. omitted) after the test for overall parallel assumption at 0.05 significance indicated that the overall parallel assumption has not been violated. In all analyses, sampling weights are used to account for the complex sampling design and to allow inferences valid for the population. A 2-tailed $\mathrm{p}<0.05$ is considered significant.

\section{Discussion}

The new infectious disease, COVID-19, pandemic affects many aspects of human life including socioeconomic, health, nutrition and food security (13-16). Proper nutrition is essential for immune system to function properly against pathogens including coronavirus (17). However, a balanced diet might be a casualty in COVID19 epidemic (18). A study in south Africa reported COVID-19 lockdown-induced changes in food access is a threat to food security in low-income households (19). Along the same line, a survey from India documented a higher occurrence rates of COVID-19 in the states with high prevalence of underweight and anemia (20).

Because of pandemic lockdown and consequent economic changes, it is expected that expensive food items, notably animal protein sources including meat, fish and poultry, to be less consumed. Be that as it may, the important questions are firstly what are the alternatives of these food items? and secondly what are the potential health consequences of these dietary shifts? This national survey will clarify the answers.

\section{Financial disclosure}

The authors declared no financial interest.

\section{References}

1. Cevik M, Kuppalli K, Kindrachuk J, Peiris M. Virology, transmission, and pathogenesis of SARS-CoV-2. bmj. $2020 ; 371$.

2. Ghadir MR, Ebrazeh A, Khodadadi J, Zamanlu M, Shams S, Nasiri M, et al. The COVID-19 Outbreak in Iran; The First Patient with a Definite Diagnosis. Arch Iran Med. 2020 2020/7/1;23(7):503-4.
3. Pourmalek F, Hemami MR, Janani L, Moradi-Lakeh M. Rapid review of COVID-19 epidemic estimation studies for Iran. BMC public health. 2021;21(1):1-30.

4. Venkatesan P. COVID-19 in Iran: round 2. The Lancet Infectious Diseases. 2020;20(7):784.

5. Atalan A. Is the lockdown important to prevent the COVID19 pandemic? Effects on psychology, environment and economy-perspective. Annals of medicine and surgery. 2020;56:38-42.

6. Asahi K, Undurraga EA, Valdés R, Wagner R. The effect of COVID-19 on the economy: Evidence from an early adopter of localized lockdowns. Journal of global health. 2021 Jan 16;11:05002.

7. Lo YT, Chang YH, Lee MS, Wahlqvist ML. Health and nutrition economics: diet costs are associated with diet quality. Asia Pacific journal of clinical nutrition. 2009;18(4):598-604.

8. French SA, Tangney CC, Crane MM, Wang Y, Appelhans BM. Nutrition quality of food purchases varies by household income: the SHoPPER study. BMC Public Health. 2019;19(1):231.

9. Muhammad A, D'Souza A, Meade B, Micha R, Mozaffarian D. How income and food prices influence global dietary intakes by age and sex: evidence from 164 countries. BMJ global health. 2017;2(3):e000184.

10. Laguna L, Fiszman S, Puerta P, Chaya C, Tárrega A. The impact of COVID-19 lockdown on food priorities. Results from a preliminary study using social media and an online survey with Spanish consumers. Food quality and preference. 2020;86:104028.

11. Bracale R, Vaccaro CM. Changes in food choice following restrictive measures due to COVID-19. Nutrition, Metabolism and Cardiovascular Diseases. 2020;30(9):14236.

12. Salois MJ, Tiffin R, Balcombe KG. Impact of income on nutrient intakes: implications for undernourishment and obesity. The Journal of Development Studies. 2012;48(12):1716-30.

13. Supady A. Consequences of the coronavirus pandemic for global health research and practice. Journal of global health. 2020;10(1):010366-.

14. Nicola M, Alsafi Z, Sohrabi C, Kerwan A, Al-Jabir A, Iosifidis $\mathrm{C}$, et al. The socio-economic implications of the coronavirus pandemic (COVID-19): A review. International journal of surgery (London, England). 2020;78:185-93.

15. Laborde D, Martin W, Swinnen J, Vos R. COVID-19 risks to global food security. Science. 2020;369(6503):500-2.

16. O'Hara S, Toussaint EC. Food access in crisis: Food security and COVID-19. Ecological Economics. 2021;180:106859.

17. Calder PC. Nutrition, immunity and COVID-19. BMJ Nutr Prev Health. 2020;3(1):74-92.

18. Jayawardena R, Misra A. Balanced diet is a major casualty in COVID-19. Diabetes Metab Syndr. 2020 SepOct;14(5):1085-6.

19. Arndt C, Davies R, Gabriel S, Harris L, Makrelov K, Robinson S, et al. COVID-19 lockdowns, income distribution, and food security: An analysis for South Africa. Global Food Security. 2020;26:100410.

20. Das A, Das M, Ghosh S. Impact of nutritional status and anemia on COVID-19: Is it a public health concern? Evidence from National Family Health Survey-4 (20152016), India. Public Health. 2020 Aug;185:93-4. 